\title{
Événements, discours, médias : réflexions à partir de quelques travaux récents
}

Event, Discourse, Media: Reflections on Recent Research

\section{Lorella Sini}

\section{(2) OpenEdition \\ Journals}

Electronic version

URL: http://journals.openedition.org/aad/1912

DOI: 10.4000/aad.1912

ISSN: 1565-8961

Publisher

Université de Tel-Aviv

\section{Electronic reference}

Lorella Sini, «Événements, discours, médias : réflexions à partir de quelques travaux récents », Argumentation et Analyse du Discours [Online], 14 | 2015, Online since 09 April 2015, connection on 23 September 2019. URL : http://journals.openedition.org/aad/1912 ; DOI : 10.4000/aad.1912

This text was automatically generated on 23 September 2019.

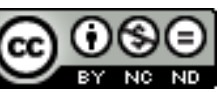

Argumentation \& analyse du discours est mis à disposition selon les termes de la licence Creative Commons Attribution - Pas d'Utilisation Commerciale - Pas de Modification 4.0 International. 


\section{Événements, discours, médias : réflexions à partir de quelques travaux récents}

Event, Discourse, Media: Reflections on Recent Research

Lorella Sini

Personne ne sait ce qui se passe aujourd'hui parce que personne ne veut qu'il se passe quelque chose. En réalité, on ne sait jamais ce qu'il se passe; on sait seulement ce que l'on veut qu'il se passe, et c'est comme ça que les choses arrivent.

(Réplique de Jean-Pierre Léaud dans La naissance de l'amour de Philippe Garel, 1993)

On entend souvent répéter ce lieu commun teinté de polémique : les médias «créent l'événement ». Celui-ci naîtrait donc dans les discours à partir de faits d'actualité bruts dont un regard objectif pourrait peut-être rendre compte si les journalistes ou les faiseurs d'opinion ne falsifiaient pas cette réalité. Et c'est bien autour de la dualité fait / événement que s'articulent les nombreuses études pluridisciplinaires parues ces dernières années consacrées au concept d'« événement ». La recrudescence d'intérêt pour ce dernier est sans doute due à la multiplication des moyens de diffusion de l'information et à la prolifération de faits dont nous pouvons avoir connaissance mais qui risquent à tout instant d'être noyés par la rumeur du monde. Les analyses de ces récents essais ainsi que les actes d'un congrès que nous voudrions illustrer ici en particulier, se situent à la croisée des études sur les sciences de l'information et de la communication et des études de linguistique à proprement parler. Justifiant l'affirmation d'Eliseo Veron (1981) selon laquelle « les médias informatifs sont le lieu où les sociétés industrielles produisent notre réel ", ces études tentent de circonscrire le concept central d'« événement ", envisagé essentiellement dans le cadre particulier des discours de la presse écrite.

Après une brève présentation de ces travaux, nous essaierons de mettre en lumière à partir de leurs observations et analyses, le cheminement qui conduit de l'appréhension $\mathrm{du}$ fait à proprement parler, au travers de sa désignation parfois labile, jusqu'au processus de dénomination de l'événement qui le définit en tant que tel. L'événement 
reconfigure les faits, à travers les formes linguistiques de sa nomination et, comme tout processus langagier, celle-ci est le résultat de tensions discursives complexes. Nous tenterons de montrer de quelle manière cette construction doit tenir compte, tout d'abord, des règles normatives relatives à une langue particulière dans les processus de création lexico-génétique et les agencements syntagmatiques qu'elle réalise; ensuite, nous illustrerons les matrices sémantiques à proprement parler fondées sur des dynamiques interactionnelles et sociales, à la base de la production du sens des expressions désignant un événement; enfin, dans une dernière partie, nous proposerons une réflexion plus étendue sur les problématiques énonciatives ou argumentatives qui entrent en jeu dans la mise en récit de l'événement, une narration qui est aussi une mise en discours. En ce qui concerne ce dernier aspect, nous évoquerons les multiples angles d'appréhension des mécanismes langagiers à l'œuvre dans ce type d'écriture médiatique, qui auraient peut-être pu bénéficier d'une analyse plus attentive.

Ces études s'intéressent en particulier à certains événements médiatiques présents dans notre actualité mais aussi à des événements plus éloignés dans le temps, ce qui permet, par exemple, à Jocelyne Arquembourg (L'événement et les médias, 2011) de relever comment a pu se construire le premier événement médiatique moderne: le tremblement de terre de Lisbonne de 1755. Elle montre selon quelles modalités il a influé sur les systèmes interprétatifs de l'époque, faisant apparaître deux visions du monde antagonistes où s'opposent croyances religieuses et vision systémique newtonienne de la nature. La comparaison avec le traitement médiatique du tsunami de décembre 2004 en Thaïlande (ibid.: chap. 6) permet de dégager des "temporalisations différenciées » dans la mise en intrigue de l'événement, en France ou en Asie, et la manière dont a été vécue cette "traversée d'une expérience collective "; l'analyse des réseaux de relations établis entre les victimes d'un côté et les donateurs de l'autre, par l'intermédiaire des ONG, permet également d'articuler les points de jonction entre fait, événement et actions consécutives à ceux-ci (ibid. : 120).

L'essai de Laura Calabrese (L'événement en discours - Presse et mémoire sociale, 2013) étudie tout particulièrement la titraille où les "désignants d'événement » exercent "une force centripète » en articulant les notions d'énonciation et d'événement. L'analyse du discours dont elle s'inspire reprend les notions élaborées par Sophie Moirand (2007b) sur la cristallisation d'une mémoire à la fois cognitive et interdiscursive dans le « motévénement ». Après avoir parcouru les différentes approches théoriques, qu'elles soient philosophiques (Badiou, Searle), sémiotiques (Ricœur), sociologiques (Quéré), l'auteure dresse un tableau des catégorisations possibles des différentes formes de nomination et recentre donc son étude sur des paramètres linguistiques relatifs à la lexicogenèse des expressions nominales (Kleiber).

Quant à l'étude de Marie Véniard (La nomination des événements dans la presse - Essai de sémantique discursive, 2013), elle relève des «moments discursifs » dans un corpus tiré de deux quotidiens nationaux, Le Monde et Le Figaro : ces derniers surgissent à l'occasion d'événements particuliers comme la guerre en Afghanistan ou le conflit des intermittents du spectacle. Conformément au titre de son ouvrage, Veniard, s'intéresse à la dénomination et déclare se situer dans une optique sémantico-énonciative plutôt que sémantico-cognitive. Ainsi, elle tente de mettre en évidence les aspects dialogiques et plus spécifiquement certaines formes d'hétérogénéités discursives dans l'appréhension de l'objet « événement ». 
Parallèlement, un congrès franco-italien de 2011, organisé sous l'égide de l'Université Sorbonne nouvelle et de l'Université de Bologne-Forlì a également donné lieu à une publication et un numéro spécial de la revue en ligne du Département d'interprétation et de traduction de l'université italienne, Mediazioni 15, autour de ce même thème: "Dire l'événement - Langage, mémoire, société» (2013). Les nombreux articles développent les différentes facettes de l'expression de l'événement dans une transversalité disciplinaire, de la linguistique à l'Histoire. En effet, le renouveau des études sur l'événement intéresse l'historien, lequel, s'inspirant de Michel Foucault (1969), devient un historien du discours, en particulier, dans l'analyse des énoncés d'archive et de ce qu'ils révèlent d'une certaine vision du monde, ce « monde lingual » selon l'expression de Sieyès volontiers cité par Jacques Guilhaumou (2006). Pour l'historien tout autant que pour le journaliste, le temps présent «commence à se constituer comme un objet historique à part entière " (Calabrese 2013a: 27). Façonné par les acteurs sociaux et les médias qui servent de support aux représentations verbales ou sociétales, l'énoncé, d'abord fluctuant, attestant le fait puis l'événement dans un travail interprétatif "second» (ce que les journalistes appellent souvent "décryptage »), prend forme; cette transaction langagière conduit, dans l'espace public, à une configuration partagée du sens et de la référence ; il opère un certain consensus dans une communauté de parole et s'inscrit dans la châne infinie des discours dans laquelle il est pris. Identifier un événement discursif, c'est « reconstituer son cheminement ontologique, empirique et historique » (Guilhaumou $2006: 211$ ). En tant que configuration d'énoncés en devenir, l'événement constitue un objet d'étude aussi bien pour les linguistes que pour les chercheurs en sciences sociales ou les historiens.

\section{Problématiques définitoires}

La question «qu'est-ce qu'un événement? " est loin d'être anodine. Bien plus qu'un terme polysémique, le concept d'« événement » serait un impensé pour J. Arquembourg (2013: 12), un point aveugle dans l'intelligibilité de son processus. Ainsi, cette interrogation pourrait apparaître comme une sorte d'aporie, car la notion d'« événement » ne renvoie qu'à une entité ambigüe, une substance glissante, délicate à circonscrire, dans la mesure où on ne sait pas quel objet il recouvre. C'est pourquoi nous préférons, au moins dans un premier temps, adopter une démarche classiquement linguistique et prendre en considération les usages de ce vocable dans le langage ordinaire, qui témoignent de sa valeur sémantique et qui révèlent la nature publique et sociale de son sens.

Le signe «événement» peut recouvrir plusieurs emplois dans nos pratiques langagières. Il semble être un hyperonyme de "phénomène", «incident", "accident", «avènement» ou «fait» qui servent à l'occasion de reprises anaphorisantes dans les mises en récit successives d'un événement particulier au cours du temps, et avec lesquels il est en distribution complémentaire. Intuitivement, on serait induit à penser que le fait, qui se produit dans le monde réel, précède la désignation de l'événement en tant que tel, généralement par une opération de dénomination (le plus souvent un nom ou un syntagme nominal) dans le cadre d'une construction narrative: "les matérialités langagières émergent une fois que le comptage des victimes a commencé » (Calabrese 2013a : 54). 
M. Charolles et B. Lamiroy (2013) constatent que le mot «fait » s'est grammaticalisé dans des expressions telles que "en fait», «au fait», "de fait», «le fait que » qui introduisent un engagement plus ou moins affiché de l'énonciateur sur la valeur de vérité qu'il entend conférer à son dire. Dans ses fonctions de connecteur, le poids sémantique du signe "fait " s'est émoussé; néanmoins ses occurrences servent à construire la posture d'observateur «objectif » lorsque les journalistes s'efforcent de serrer au plus près l'actualité, en assurant leurs lecteurs de leur neutralité vis-à-vis de ces faits qui devraient, selon l'expression consacrée, "parler d'eux-mêmes ». Le signe «événement » est resté, quant à lui, fondamentalement référentiel (par opposition à la valeur sémantique d'un connecteur) : en effet, il «appréhende des situations comme des entités singulières et distinctes " en prenant en compte les « conséquences qui en découlent ou qui peuvent en découler ». Cette constatation semble vouloir montrer que le fait sert à désigner une entité physique brute initiale, une donnée objective préalable, antérieure à toute interprétation, "avant saisie perceptive et interprétative » (Charaudeau, cité par Arquembourg 2013: 8). Un " être-là » avec son « indéniable prégnance avec le réel [...], un idéal de langage qui serait sans émergences discursives [...], un continuum non-événementiel» (Margarito 2013). Le fait, comme pourrait l'être l'acte de marcher ou de courir, serait donc un "non-événement », un acte routinier irréfléchi, sans conséquence signifiante ou à signifier. Les faits en tant que tels "sont soustraits, tout au moins provisoirement, à l'argumentation", disait Chaim Perelman (1992: 154) : ce sont des objets à propos desquels les argumentateurs ont dissipé toute controverse et qui constituent dès lors une prémisse à tout développement argumentatif.

Par opposition, l'événement (de e-venit, ce qui vient du dehors) est ce qui fait irruption dans l'espace public. Son caractère disruptif le distingue, en effet, des activités routinières du quotidien. La dualité entre le phénoménologique et le discursif pour parler de la factualité, est la pierre d'achoppement qu'il est parfois hasardeux de circonscrire. "Le signifié d'une phrase ne peut pas être conçu comme une "image" d'une situation ou d'un événement, comme le veut la linguistique cognitive » (Prandi 2013). La question de savoir s'il existe une quelconque motivation a priori entre le type d'événement et le désignant d'événement, sous la forme d'un toponyme ou d'une date (Calabrese 2013a : 209), ne nous semble pas une démarche appropriée pour un analyste du discours qui s'attache avant tout à interpréter des textes. Elle pourrait induire à penser que le signe indexe univoquement sa chose alors que le sens naît, pour le linguiste, du tout du discours. Rien n'indique dans la forme linguistique hors contexte des expressions « Tchernobyl » ou « le 21 avril », qu'elles se réfèrent à des événements. De même, opérer une distinction entre la construction médiatique des événements et la construction de la réalité, comme semble le préconiser M. Veniard (2013: 21), impliquerait qu'il existe un lieu d'où rendre compte du monde avec une infaillible justesse, dans une transparence idéale. N'est-ce pas plutôt la complexité «des représentations régulièrement associées, sous la contrainte de la langue, au signifié d'un terme dans une culture et une société données qui constituent ce que l'on peut appeler son "contenu éidétique" "? (Quéré 1994 : 25). Les événements sont d'abord des changements existentiels avant de devenir des objets discursifs (Quéré, ibid.). Mais ce changement existentiel peut-il prendre forme s'il n'est pas parlé ? Dans le roman d'Annie Ernaux intitulé justement "L'événement ", qui raconte l'histoire intime et tragique d'un avortement, l'auteure cite une page de l'agenda de l'énonciatrice où il est écrit: «Formidable. Si seulement je n'avais pas cette RÉALITÉ dans mes reins ». 
L'artifice typographique utilisé, les lettres capitales et le soulignement, pour signifier "concrètement " un réel situé dans un au-delà de l'écriture, est significatif de l'impuissance à le faire exister, malgré la présence matérielle qu'il nous impose. Les images elles-mêmes qui veulent donner l'illusion d'enregistrer ce qu'il est advenu, exercent un pouvoir de fascination dont les médias abusent et qu'il est toujours raisonnable de démythifier : l'image en elle-même est difficilement interprétable si aucun discours n'ancre son intentionnalité, comme le montre J. Arquembourg en analysant les films amateurs du tsunami. Les images sont des représentations qui «donnent à voir un regard tout autant qu'un objet regardé » (Arquembourg 2013a : 63). Le mot et l'image sont complémentaires, de leur interaction naîtra le sens attribué par l'interprétant, en fonction de conventions socio-culturelles pré-requises.

Il existe « un point de bascule » où le référent du nom, en tant que construction et non en tant que reconnaissance, «devient plus imprécis, plus général aussi, pour ouvrir la désignation du fait à celle de l'événement, en incorporant la dimension de l'expérience vécue sur le plan collectif» (Arquembourg 2013 : 165). Ainsi, les événements abordés dans les essais que nous commentons ici ne sont pas des événements existentiels; il s'agit d'événements, objets de jugement, de pensée, de discours, des "occurrences découpées dans le flux des changements » (Quéré 2013) que les acteurs vont élaborer, " par la mise en place d'un récit collectif s'inscrivant dans les pratiques sociales, des expériences et un contexte historique, social, culturel et symbolique » (Veniard 2013 : 24).

Depuis le siècle dernier, l'acception du vocable « événement » est indissolublement liée au rôle que jouent les médias dans les sociétés occidentales. Telle insulte proférée sur un stade, habilement instrumentalisée par les journalistes à des fins commerciales, devient un événement qui ne dure parfois que le temps d'une saison footballistique (Baklouti, Richard 2013). Les petites phrases et autres dérapages verbaux souvent mis en exergue par les titres de journaux ou les flashs info, répétés à l'envi sur les chaînes de télévision ou les réseaux sociaux, tantôt décriés, tantôt ironiquement déconstruits dans les jeux de mots, alimentent la rumeur médiatique, comme nous l'ont montré les travaux de Krieg-Planque sur les formules (2009). Une polémique autour du port du voile islamique stratégiquement mis en scène sur les plateaux de télévision (Ravazzolo, 2013), un scandale politico-financier peuvent à tout moment devenir une "affaire d'État ». La prolifération des canaux d'informations fait que l'on assiste à une multitude d'événements médiatiques qui ne deviendront jamais des événements historiques : au $\mathrm{XX}^{\mathrm{e}}$ siècle, dit Pierre Nora, les médias fabriquent des "événements monstrueux » en attribuant «au discours, à la déclaration, à la conférence de presse, la solennelle efficacité du geste irréversible » (Nora 1972).

Pour les historiens, la labilité du concept d'événement se reflète, entre autres, dans l'instabilité des expressions servant à désigner ses occurrences, suivant le moment et le lieu où se situe l'observateur (Veniard 2013 : 28). Inscrites dans l'espace-temps, elles sont prises dans des réseaux de communication où interagissent des sujets historiquement, socialement et culturellement déterminés (Branca-Rosoff et al. 1995). "Les événements d'Algérie " ont finalement été perçus comme une "guerre", l'épisode appelé "Invasions barbares" dans nos livres d'histoire sont considérées comme "Migration des peuples" par les Allemands (Völkerwanderung). Plus près de nous, l'appellation française "Révolution de Jasmin» considérée comme trop pittoresque a été contestée par les Tunisiens (Azouzi 2013). Cela veut-il dire que les deux lexies concurrentes se disputent un même référent? Nous répondons par la 
négative car il faudra bien admettre que les synonymes absolus n'existent pas et que leurs usages en contexte expriment des sens différents; l'analyse des formations discursives comme objet de l'histoire, sont constitutives d'une formation idéologique, en accord avec des pratiques sociales différentes (Foucault 1969).

Mais, ce qu'on appelle "événement " peut également revêtir un statut singulier : hors de l'expérience, il peut être invisible dans le temps où il se produit et poser un problème d'appréhension à l'historien qui doit analyser ses représentations au moment où il s'est révélé (Laborie 2011b). E. Puccinelli Orlandi (2013) relate comment un documentaire sous la dictature argentine produit un événement sans le représenter tout en se constituant comme objet mémorial. Paradoxalement, le silence peut dessiner en creux un événement tu ou censuré; le déni de dénomination constitue alors une béance dans le fil des discours qui peut néanmoins imposer à la postérité tous ses effets de sens. Nous pensons également à la série documentaire-événement, Shoah, de Claude Lanzmann qui s'interdit de montrer les lieux des faits ou, pire, de les reconstituer par l'image; les scènes filmiques nous accompagnent dans les interminables silences des protagonistes où se loge sans doute l'inouïe vérité. Ce temps suspendu, inaudible, nonverbal qu'il a été impossible de traduire ou d'interpréter au procès Eichmann (Ballardini 2013), la mise sous silence des faits, les oublis volontaires ou inconscients, n'empêchent pas l'enchaînement des réactions qui se répercutent dans l'espace public.

C'est pourquoi la définition de l'événement doit intégrer une dimension pragmatique dans la mesure où sa configuration entraine "une praxéologie déterminée par des pratiques institutionnelles " (Calabrese 2013a: 52) : l'«acte isolé d'un loup solitaire » n'appelle pas les mêmes réactions (policières, judiciaires, etc.) qu'un " acte terroriste ". $E t$, en effet, ceci nous permet d'expliquer les raisons pour lesquelles la première guerre du Golfe a pu être désignée par certains intellectuels français de l'époque comme un " non-événement ». Peut-être voulaient-ils signifier par là qu'il ne s'agissait que d'une guerre "virtuelle", aux frappes " chirurgicales ", en somme ce qu'ils jugeaient n'être qu'un succédané de guerre. Mais ne voulait-on pas également sous-entendre que les acteurs sociaux et les institutions politiques n'avaient pas de prise sur des faits, pourtant bel et bien réels, et qu'ils n'avaient pas été symboliquement élaborés? L'Histoire est faite de récits qui énoncent l'action en même temps qu'ils la produisent. Pour que le fait se transmute en événement, il faut qu'une dynamique événementielle soit enclenchée ; ce processus, appelé "événementialisation ", construit une identité sociale en conférant au fait singulier des significations collectives qui le sémiotisent, en quelque sorte, lui conférant par là-même le statut de fait institutionnel et politique.

Deux jours après les attentats terroristes à Paris du 7 janvier 2015, un journal (Rue89) donne la parole à ses lecteurs, dans une rubrique intitulée : "Charlie : vos tribunes et poèmes qui aident à penser l'événement ». Aurait-on pu écrire : « vos tribunes et poèmes aident à penser les faits "? Ce dernier énoncé est, pour nous, à la limite de l'acceptabilité car il semble bien que le vocable « fait » n'implique pas en soi, dans son usage linguistique habituel, de fonction agentive contrairement au vocable " événement ». C'est bien, en effet, cette fonction agentive, celle à laquelle on attribue l'agir et le pâtir, qui est déterminante pour circonscrire la désignation d'un fait comme événement, puis selon des modalités contingentes plus précises, comme "crise", " guerre ", " catastrophe ", "massacre ", etc. C'est pourquoi, J. Arquembourg utilise, dans son essai, le concept d'« advenant individuel » et d'« advenant collectif » : ce sujet n'est pas un sujet autarcique doté d'une identité mais un sujet traversé par 
l'expérience ; il est défini par sa "passibilité », « une manière de répondre de soi et de ce qui arrive à travers l'appropriation des possibles ouverts par l'événement ». Le sujet collectif, quant à lui, se construit malgré la diversité des " vécus ", en réactivant et en reconfigurant le sentiment d'appartenance nationale (Arquembourg ibid.: 29-30). Pascale Goetschel et Christophe Granger (2011a : 18) ont essayé de circonscrire ce que peuvent être des "politiques événementielles", souvent réglées par de véritables protocoles (ceux des communicants en tous genres), qui participent à plein titre à "l'économie symbolique du pouvoir»: "par la magie de la foule assemblée, [l'événement] peut en effet servir à mobiliser, à aiguillonner le sentiment d'appartenance, à politiser les participants, voire à incarner l'idéal de citoyenneté ».

Enfin, l'événement se présente comme ayant à la fois des bornes spatiales et temporelles. «La sémiotique de l'événement se fonde sur une sémiotique politique de la spatialité » (Lamizet 2013) ; une déclaration politique à Bruxelles sur l'économie de la Grèce aura des conséquences sur les marchés financiers de toute l'Europe. L'espacetemps qui s'exprime en rapport avec le sujet de l'énonciation, est inscrit dans les formes linguistiques mêmes de nos langues indo-européennes: le présent de l'actualisation empathique, le passé de la distanciation, le futur ou le conditionnel de l'imagination ou de la volonté. Il est intéressant de remarquer, par exemple, comment l'oscillation entre les valeurs radicales et les valeurs épistémiques du verbe modal "pouvoir» expriment, dans certains genres discursifs particuliers - comme par exemple le "rapport éducatif » (Sitri 2013) -, une transformation du sujet: dans « X a pu dire ou faire... », on entend "X est désormais en capacité de... ». La teneur d'un événement se saisit au travers de ses conséquences factuelles et des résonances qu'il crée auprès des acteurs sociaux. Il provoque des réactions souvent inattendues et imprédictibles auprès de l'opinion publique, à commencer par les journalistes ou les historiens eux-mêmes et leurs auditeurs-lecteurs, en passant par les décideurs politiques. Les causes de l'événement émergent également a posteriori au travers d'indices avant-coureurs qui acquièrent rétrospectivement un sens, une directionnalité : "de contingent a priori, l'événement devient nécessaire a posteriori » (Arquembourg 2013:21). Cette réflexion recoupe celle de Quéré qui pose le caractère téléologique du processus événementiel : en tissant la trame des événements entre eux, ce processus se déploie selon un "devenir orienté ». Nous sommes des "prophètes tournés vers le passé » (Arendt, citée par Quéré 2006). L'événement existentiel se convertit alors en objet de pensée et de jugement, il acquiert une signification provisoirement inachevée par l'appréhension de ses causes et de ses conséquences possibles : il revêt alors un pouvoir herméneutique (Quéré 2006, 2013).

Reprenant les différents parcours conceptuels précédemment élaborés par ses précurseurs, Laura Calabrese tente une définition exhaustive de l'événement médiatique :

Qu'il s'agisse de "construction», de "constitution», de "mise en forme» ou encore de "préfiguration ", l'événement est soumis à un processus de mise en sens par les médias, parmi d'autres institutions sociales qui y participent ultérieurement dans une plus ou moins grande mesure (l'école ou l'université par exemple, mais aussi des collectifs politiques ou des acteurs sociaux moins consensuels). L'événement médiatique, loin d'être un produit original du méta-énonciateur, se construit selon des normes collectives, à partir d'un stock social des connaissances et en fonction de scripts façonnés par les imaginaires professionnels qui anticipent les attentes du public (Calabrese 2013a : 114-115). 
Résultat d'une pratique sociale et discursive, l'événement médiatique est une représentation dynamique, produite collectivement dans la cité par un certain consensus; son expression reflète les habitus linguistiques de tel ou tel organe de presse, des formulations en partie prévisibles mais que l'auditoire peut toujours à son tour remodeler.

\section{De la dénomination à la narration}

Dans ses manifestations linguistiques, l'événement est appréhendé par sa dénomination, ses valeurs sémantico-discursives et sa mise en récit. Nous pourrions proposer d'évaluer ces différents aspects, qu'ils soient lexicologiques, syntaxiques, sémantiques ou discursifs, selon une progression dans l'analyse de la représentation de l'événement, tout en considérant que ces paliers d'appréhension sont interdépendants l'un de l'autre.

Le choix du corpus d'observation oriente la description et l'analyse du linguiste. Ainsi L. Calabrese s'intéresse particulièrement à la titraille où les "désignants d'événement " sont plus facilement circonscrits, tandis que Veniard, grâce au logiciel de statistiques textuelles Lexico 3, balise son corpus autour de certains mots-pivots comme "Afghanistan» ou «intermittents». C'est ainsi qu'apparaissent des concrétions syntagmatiques correspondant à des configurations événementielles. Cette objectivation d'entités discrètes qui prend la forme d'un nom ou d'une lexie est rendue nécessaire dans la mise en récit. Arquembourg, quant à elle, a analysé un corpus de presse et les modalités des débats publics que tremblement de terre et tsunami ont suscitées, ce qui, de fait, place son étude dans le champ plus large de la narration ou des discours et de leur réception.

\subsection{Processus lexicologiques et néologiques relatifs à la dénomination}

C'est Veniard, conformément au titre de son essai, qui aborde plus spécifiquement les processus lexicologiques qui régissent l'acte de nomination. Pour ce qui est de l'événement, cet acte initial peut s'apparenter à un acte de baptême : en se stabilisant, la dénomination devient propre à la désignation d'un événement particulier et lui assure sa rémanence dans la mémoire collective. Du point de vue formel, ce désignant d'événement (comme l'appelle Calabrese) est typiquement un nom (souvent un déverbal) ou un syntagme nominal dénotant une action. "L'homme au cœur greffé " devient «la greffe du cœur» (Calabrese 2013a: 112), «Jaurès est mort» devient quelques jours plus tard «la mort de Jaurès ». Cependant la morphologie lexicale des langues particulières exerce une contrainte sur la lexicogenèse des substantifs en question: alors même que le verbe "intransiger» n'existe pas, on parlera de "l'intransigeance du gouvernement»; inversement la "transigeance» n'est pas attestée dans la langue française même si le verbe "transiger" fait partie de son système. Ainsi, ces dénominations différentielles ne sont pas toujours justifiables dans l'absolu: la période appelée «entre-deux-guerres» est connue en Italie sous le chrononyme il Ventennio («les vingt années»). Même s'il est inconfortable de l'admettre, la part de hasard dans la nomination est constitutive de l'activité créatrice en linguistique, ce que ces études semblent écarter. Bien au-delà du «caractère labile 
de l'association entre un nom et une information événementielle " (Calabrese 2013a: 151), c'est du bien-fondé de l'arbitraire du signe dont il faut se persuader, «de [sa] présence [...], de sa modification, plus ou moins grande après un temps, ou de son annihilation après un autre temps ", disait si bien Saussure (2002:68).

La dénomination d'un événement est le résultat d'une opération d'aspectualisation au sens linguistique du terme : on "déclenche une grève » (aspect ponctuel et inchoatif du sémantisme verbal), alors qu'on dira "la manifestation a duré toute l'après-midi " (aspect duratif). À ce sujet, Calabrese (2013a : 148) cite les études de Kiefer (1998) selon lequel l'aspect fait partie des " programmes de sens ", c'est-à-dire des traits capitalisés constitutifs des dénominations. Or, pour nous, l'expression "programme de sens » pourrait donner à penser que le sens actualisé par les noms d'événement est potentiellement inscrit dans leur forme initiale, qu'il pourrait être déterminé de manière rationaliste ou substantialiste une fois pour toutes. Rien n'indique a priori dans la nature des vocables " séisme ", « cérémonie » ou " guerre », en dehors de ce que Paul Siblot appelle leur " praxis » (2001), leur identité qui est modelée par les usages qu'une communauté de parole peut en faire (par exemple dans l'ensemble ouvert des usages dits dérivés ou métaphoriques de ces vocables, difficilement prédictibles). Pourquoi, par exemple, ne pas proposer une perspective constructiviste, pour laquelle « une séquence ne donne lieu à un énoncé bien formé qu'eu égard à un contexte qu'elle détermine étroitement » (Franckel 2002:10) ? L'appréhension de l'événement est liée à la saisie cognitive particulière de l'observateur en un moment donné de son énonciation; il s'exprime selon qu'il envisage le processus de l'événementialisation, en fonction d'angles particuliers de la saisie du temps qui n'est pas intrinsèquement impliquée par le sémantisme des lexèmes mais appréhendée par l'ensemble de l'expression immergée dans son contexte discursif: par exemple la durée ("Euthanasie: l'éternel débat»), la sporadicité («Le phénomène des "voisins vigilants" »), l'itérativité (tous les faits que l'on peut regrouper sous l'appellation de la «loi des séries»: «Accidents aériens à répétition»), le résultatif («la chute du gouvernement »), ou encore la visée : on voit, par exemple, comment l'événement discursif «passage à l'euro » a d'abord précédé puis accompagné l'instauration de la monnaie unique européenne (Modena 2013). Toutes ces dénominations montrent aussi bien l'objet désigné que la position subjective adoptée par le locuteur pour le nommer (Siblot, cité par Veniard $2013: 145$ ).

Du point de vue lexical, le nom d'événement peut être érigé en nom propre lorsque sa saillance devient importante au point de devenir un «désignateur rigide (Kripke 1980), un signe monoréférentiel. Le désignant d'événement parfois affublé d'une majuscule est alors assimilable à une expression figée: ainsi, le nom propre d'événement peut être de manière métonymique ou par antonomase, un toponyme («Tchernobyl»), un phénonyme (un événement météorologique ou naturel : «Katrina»). Calabrese (2008) propose de distinguer les chrononymes (nom de période historique : «le Paléolithique») des héméronymes (une date: «le 11 septembre »); mais nous pouvons également regrouper l'ensemble des événements historiques sous le terme de "praxonymes " («La Résistance», « la guerre de trente ans ») qui indique que le sens du nom propre renvoie toujours à des pratiques sociales. En réalité, ces classifications pourraient être multipliées, tant il est vrai que l'identification de l'objet, prend de plus en plus souvent, dans nos sociétés complexes, la forme d'une dénomination propriale (nom-name). Ainsi, le nom du chalutier coulé, le «Bugaled-Breizh», un "chrématonyme» selon la terminologie germanique, sert 
métonymiquement à dénommer l'affaire du même nom. On trouve par exemple dans Le Télégramme daté du 31 mai 2014, le titre suivant: «Bugaled-Breizh : autopsie d'un non-lieu ».

La création des noms d'événement relève, de fait, de processus néologiques classiques. Tel est le cas, par exemple, du recours à l'emprunt exogène pour référer une réalité présentée de manière emphatique : l'impact du vocable «tsunami » est plus percutant que son équivalent français «raz-de-marée» et il permet de plus de situer la catastrophe en un lieu éloigné, donc en quelque sorte de neutraliser sa dangerosité. L'introduction des xénismes dans les textes d'actualité est mimétique du transfert spatial que l'énonciateur opère lorsqu'il se place en esprit dans une réalité étrangère ; ce sont les realia (comme "Intifada») qui résistent à l'opération traductive et qui contribuent à la représentation im-médiate, donc plus "vraie", d'un événement univoquement circonscrit dans un espace géographique et historique. L'intégration de ces vocables dans le langage ordinaire, leur « migration de signification " comme dirait Saussure, ou les parcours entre deux champs discursifs " colingues » (Branca-Rosoff, Guilhaumou 1998) peut être, malgré tout, très rapide même si leur rentabilité par des formes dérivationnelles est très faible: «intifada» a pu servir, à l'occasion, de synonyme de «révolte des banlieues" mais peut difficilement supporter un affixe (même s'il n'est pas impossible que "néo-intifada" ne soit attesté un jour); "Watergate", au contraire, s'est décliné suivant de nombreux néologismes dans le langage ordinaire : « Irangate », « Monicagate » etc...

\subsection{Processus syntaxiques et syntagmatiques}

L'analyse syntaxique et syntagmatique nous montre en quoi une langue particulière nous oblige à dire certaines choses selon des modalités - par exemple les positions syntagmatiques des unités phrastiques - dictées par la norme d'un système linguistique donné, en étroite relation avec un genre discursif particulier. L'étude de ces agencements syntaxiques à propos des dénominations d'événements a été menée à partir de l'observation des titres. La lexicométrie fournit à l'analyste du discours un outil révélateur des combinatoires préférentielles de certaines unités linguistiques - le mot entouré de son cotexte -, avec une relative exhaustivité et systématicité : Veniard relève ainsi de manière privilégiée les syntagmes prépositionnels (sous la forme Syntagme Nominal - Préposition - Syntagme nominal) comme «la guerre contre l'Afghanistan ", et «le conflit des intermittents » (2013: 85-6). Grâce à la constitution d'un corpus, l'observateur relève un "profil lexico-discursif» qui «rassemble les caractéristiques préférentielles de la combinatoire et du fonctionnement discursif de ce mot » sur les plans syntaxique ou syntagmatique, par le repérage des cooccurrences, des collocations, du cotexte (Veniard 2013: 55). L'emploi des articles ou leur absence dénote une évolution perceptive et une accession à une présence plus prégnante du fait dans l'actualité (Kleiber 1983, cité par Calabrese 2013a: 155). Passer de « Un attentat s'est produit à Karachi » à «L'attentat de Karachi », puis à "Attentat de Karachi » accompagne cette évolution vers une saisie cognitive du référent. Celle-ci devient de plus en plus abstraite suivant son degré d'accessibilité dans la mémoire à plus ou moins long terme du récepteur. D'autres matrices syntaxiques ont été relevées par L. Calabrese (2013a : 106) comme la formation typiquement bi-segmentale thèmerhème dans les titres: "URSS: agitation dans les Républiques». On aurait pu également remarquer que la formation des composés syntagmatiques est soumise à des 
pressions synthétiques relevées pour le français, entre autres, par Picone (1991) : on dira «l'affaire Gregory » ou "le procès Erika » où les composés ont effacé le joncteur « de ». De même, la position et la fonction de l'adjectif ou de l'adverbe dans le syntagme nominal auraient peut-être mérité une observation plus attentive. Ainsi, un livret du journal Le Monde consacré au style de ce quotidien (2002) distinguait, de manière normative, les titres manchette, les titres informatifs, les titres incitatifs ou les titres commentaires dans lesquels la présence ou l'absence du verbe devait assumer un rôle spécifique dans la visée de l'article; la présentation d'un événement peut se mouler suivant le moment ou la modalité de sa réception dans l'une ou l'autre de ces formes.

\subsection{Problématiques autour de la construction du sens}

Après une certaine hésitation, la dénomination finit par être généralement admise dans la langue par la collectivité, même si à une certaine " déférence » (Calabrese 2013b) vis-à-vis de ce que les théories de l'argumentation appellent l'argument d'autorité l'opinion publique peut toujours opposer un contre-discours. Les débats autour de la dénomination de la Révolution de jasmin ou de la guerre d'Algérie révèlent des enjeux éthiques et traduisent une "inquiétude» au regard du discours (Foucault 1971). La saillance de ces noms d'événement apparaît au cours de la «configuration » des faits par les médias - terme préféré à « construction » par M. Veniard -, en particulier dans les titres, les annonces, les brèves défilant en bas d'un écran de télévision, dont la forme est contrainte par les nécessités techniques. Mais l'émetteur se doit aussi de tenir compte plus trivialement des stratégies mnésiques, de captation du lecteur ou de guidage dans le balayage synoptique de la page (Charaudeau 2005, Douël 1987, cité par Calabrese 2013a : 102). Le manque d'espace et l'économie de la production des réseaux de communication favorisent les phénomènes de surassertion ou d'aphorisation (Maingueneau 2006). Le mot déploie un profil interdiscursif qui « traduit et conditionne l'expérience qu'une communauté de locuteurs fait d'un objet du monde » (Veniard 2013 : 55). Certains mots peuvent être considérés comme des mots-arguments, c'est-àdire qu'en acquérant une indexicalité événementielle, ils sont le dépôt de la mémoire d'une communauté langagière (Moirand 2007a); en effet, ces unités linguistiques finissent par fonctionner sous le régime de l'allusion plutôt que celui de la désignation (Moirand, citée par Calabrese 2013a: 90, 228). Ainsi, la traduction de l'expression américaine "war on/against terror " a été traduite en français par "guerre contre le terrorisme " et non pas "guerre contre la terreur ", car «terreur » évoque pour les francophones la période (post-)révolutionnaire (Veniard $2013: 108$ ). Le mot «lutte » et le mot « conflit » relevés respectivement dans Le Monde et le Figaro pour parler de la grève des intermittents ne déploient pas la même potentialité idéologique, n'actualisent pas les mêmes scénarios argumentatifs ou topoï. Les discours ne sont pas seulement le reflet des tensions sociales, ils les constituent bel et bien (Foucault 1969, Faye 1972, Guilhaumou 2006). C'est particulièrement évident lorsqu'il s'agit de statuer sur l'introduction d'un terme dans les textes qui ont une portée politique; les paradigmes désignationnels se construisent selon une dynamique interdiscursive, et remplacer le terme "parité " par "égalité » engage des actions citoyennes dont la teneur est loin d'être négligeable pour les intéressées (Raus 2013).

En quelque sorte, après s'être construite syntaxiquement selon la norme linguistique d'une langue donnée, une expression déterminée s'inscrit dans le discours avec ses propres habitus (au sens bourdieusien) relatifs à une langue-culture déterminée dont 
nous n'avons pas toujours conscience. Elle se présente en combinaison préférentielle avec certains lexèmes (dont de véritables "clichés »), et elle implicite ce que nous pourrions appeler un même moule argumentatif (un topos) en fonction des opinions. Parler de "guerre en Afghanistan ", de "guerre contre le terrorisme » ou de "guerre contre Al-Qaïda » ne convoque pas et ne reflète pas le même point de vue sur ce conflit. Matrices sémantiques, formules, patrons formulaires, routines journalistiques sont autant de modalités qui constituent une sorte de canevas à partir duquel l'événement est mis en scène. Les titres du journal Libération et leurs célèbres formes paronymiques (" À la gare comme à la guerre » chapeautant un article sur la grève des cheminots) ont assuré à ce journal une large audience. À ces créations ludiques, il faudrait ajouter un paramètre peu étudié car difficilement systématisable mais dont la pertinence est pourtant manifeste dans l'expression linguistique: la prosodie sémantique (Sinclair 2004, cité par Veniard 2013 : 53). L'ordre des séquences, comme dans cette construction symétrique binaire: "Bush mobilise, les Bourses plongent", où les assonances sont évocatrices, donne corps au rythme de la phrase. Celui-ci est aussi pertinent dans la lecture-écriture d'un poème que dans la lecture-écriture d'un énoncé du langage ordinaire.

\subsection{La mise en récit de l'événement et la pragmatique du texte}

L'actualité prend forme au moment où elle fait émerger l'événement, par la construction d'un récit. Les mécanismes sémiotiques qui sont à l'origine de la composition du récit d'un événement réel ou fictif, sa narration orale ou écrite, puis sa réception et sa compréhension par ceux à qui elle est destinée, ou par les chercheurs qui l'analyseront parfois des générations plus tard, ont été définis aussi bien par les historiens (Nora) que par les philosophes (Gadamer), les théoriciens de la littérature (Genette, Ricœur), les traductologues (Meshonnic, Steiner), les linguistes et autres sémioticiens (Greimas, Barthes). Ayant dépassé la froide mécanique de l'approche structuraliste et "s'affranchissant de sa clôture sémiotique » (Arquembourg $2013: 38$ ) encore qu'il faudrait suivre plus attentivement les spires de la pensée théorique saussurienne -, l'analyse linguistique semble être le présupposé indispensable à une théorie de la compréhension c'est-à-dire à une herméneutique. J. Guilhaumou, par exemple, admet le tournant linguistique des études historiques, en partie grâce aux progrès qu'a permis l'analyse numérique des corpus. Cette herméneutique étudie le sens de documents qui doivent être lus à travers le filtre temporel de l'Histoire et grâce à la participation du chercheur lui-même en tant que sujet historique. «Quelle réalité matérielle l'histoire a-t-elle en dehors du langage, en dehors de notre croyance interprétative en des données enregistrées essentiellement en termes linguistiques?» (Steiner $1978: 29$ )

L'immédiateté de l'actualité impose une certaine forme au récit quasi-synchronique qui se distingue de sa mise en discours à proprement parler. Ce discours reconfigure les faits selon un éclairage particulier que le temps et la société des hommes lui ont conféré. C'est pourquoi, c'est le rapport chronologique des faits par des témoins oculaires qui l'emporte dans un premier temps, aussi bien dans le reportage du tremblement de terre de Lisbonne que dans celui du tsunami, ou dans le conflit des intermittents du spectacle. On a coutume d'évoquer la routine professionnelle de la règle des $5 \mathrm{~W}$, préconisée dans les Écoles de journalisme pour guider la rédaction des titres et des articles de presse: Who?, What ?, Where?, When ?, Why ? (Calabrese 2013a : 
103 ; Arquembourg 2013 : 35). Mais les contraintes économiques qui doivent faciliter l'accès à une information aussi simple, concise et lisible que possible ne neutralisent pas la présence de l'énonciateur dont le message garde toujours une empreinte. Selon que l'on relate les faits pris sur le vif ou qu'on apporte des éléments d'analyse avec le concours des acteurs sociaux qui les appréhendent dans un second temps pour construire la perception de l'événement en tant que tel, les scénarios énonciatifs diffèrent. Il faudrait poser ici la question du genre de discours dans lequel s'insère ce type de récit, identifier les différences linguistiques, par exemple, entre la représentation de l'événement dans un témoignage oculaire et dans le rapport d'un envoyé spécial sur les lieux des faits et, accessoirement, démontrer en quoi ces deux types de récits s'imbriquent ou s'ils se départissent l'un de l'autre. Intuitivement nous serions enclins à penser que monde raconté et monde commenté, les deux régimes énonciatifs illustrés par Benveniste, servent et en même temps convoquent respectivement l'attestation des faits et la construction de l'événement. Mais il s'agirait de vérifier, dès la formulation du titre, dans les marques aspecto-temporelles, axiologiques, l'usage de la deixis, des connecteurs, de la thématisation, dans les variations de registre etc., tous ces indices d'une subjectivité plus ou moins contrôlée ou assumée par le journaliste ou par l'auteur des documents-source qu'un analyste du discours est à même de recueillir. Les formes narratives relatant des faits et/ou un événement, dévoilent tout un éventail d'implicites ou de présupposés qui devraient permettre de distinguer le dire du dit, pour reprendre la formule célèbre de Ducrot, voire de relever "l'évanouissement narratif de l'objet », les modulations discursives qui permettent de signifier ce qu'on ne peut pas dire vraiment (Jamet 2004). Et si l'on admet que tout énoncé s'adressant à un auditoire qu'il s'agit de séduire, de persuader ou de convaincre, entraîne des réactions conséquentes, qu'il donne lieu à des questions alimentant les débats publics, alors tout acte énonciatif qui conduit au bout du compte à la dénomination d'un événement est un acte performatif : en ce sens, tout énoncé recèle des intentions argumentatives (Amossy 2000).

Rapporter des faits, cela sous-entend pour un journaliste que l'on évitera tous les indices susceptibles de trahir une émotion ou pire, une opinion politique. Mais, comme le démontre Koren (1996), cette posture éthique révèle une position idéaliste (dans tous les sens de ce terme) qui, faisant abstraction d'une "médiation" linguistique, s'exprimerait comme dans un "miroir social». Or cette médiation, malgré les « effets d'objectivité » présents dans l'écriture de presse, est constitutive de la prise de parole. Le journaliste participe sur le terrain à l'Histoire en train de se faire avec ses préjugés légitimes, ses représentations stéréotypées, ses clichés verbaux (ceux que l'on s'efforce justement de combattre dans les Écoles de journalisme) ; il se fait occasionnellement le porte-parole d'une cause humanitaire, il est parfois victime de prises d'otage largement médiatisées. Sa présence sur la scène publique, ses commentaires "de surplomb », son phrasé trahissent donc un ethos (ce concept n'est jamais pris en compte dans ces études), partie prenante du sens véhiculé par un énoncé. Koren (1996) pose une question à ses yeux essentielle à l'instauration d'un débat véritablement démocratique : le journaliste ne doit-il pas assumer publiquement ses propres prises de position politiques?

Le discours journalistique est, par ailleurs, par essence polyphonique, marqué par l'éclatement des sources énonciatives: le médiaphorique ou évidentiel (Hagège 1995 ; Rabattel 2006) se manifeste dans le discours par des traces d'hétérogénéité discursive montrée ou constitutive par lesquelles on accorde à l'information un degré plus ou 
moins assumé de fiabilité (Authiez-Revuz, cité par Veniard: 68): «c'est par la monstration de l'autre dans le dire que l'identification idéologique [d'une réalité sociale imaginaire] se réalise » dit Hailon à propos de l'usage autonymique du terme «voyoucratie» (2013: 263). La mémoire des mots, leur fonction citative ou " particitative » (Maingueneau 2004), la connivence présupposée entre le journaliste et son lecteur est particulièrement délicate à détecter par les logiciels : pour donner un exemple récent, les désignations du califat/Etat islamique par les différents acronymes « ISIS », « DAESH », voire par le sigle « EI » ne sont pas des actes neutres ; écrire avec ou sans guillemets, avec ou sans point d'interrogation les énoncés «complot judéomaçonnique (?) » ou « attentat islamo-fasciste (?)» (dans les titres commentant les attentats de janvier 2015 à Paris) dénote des « isotopies antagonistes » construites selon une certaine doxa qu'il s'agit aussi d'interpréter (Amossy 2014).

\section{Conclusion}

Les études dont nous avons rendu compte ici repoussent unanimement l'idée que l'on puisse reconstruire la vérité des faits, malgré la prétention d'objectivité affichée encore parfois par les journalistes. La difficulté à saisir l'objet « événement » provient de cette oscillation de l'observateur, sujet social et historique, tout à la fois tenté de matérialiser l'existant d'un état de choses et contraint de l'exprimer par les formes linguistiques particulières de sa langue, qui l'obligent à façonner ses dires dans des moules syntaxiques, sémantiques, mais aussi argumentatifs au sens large. Ces travaux ont mis en lumière de manière souvent très détaillée, grâce aussi à des indices quantitatifs, les relations empiriques que les manifestations langagières relatives à la dénomination de l'événement, puis à sa mise en intrigue, entretiennent avec la réalité sociale qui l'accueille et l'interprète par des actions publiques, voire politiques. La problématique philosophique autour des processus de production du sens continue d'alimenter les débats dans ses approches linguistiques, sociologiques, historicistes. L'intelligibilité de l'événement, surtout lorsqu'il surgit sur la scène de l'actualité en train de se faire, ne peut faire abstraction, selon nous, d'une analyse plus poussée des visées intentionnelles plus ou moins assumées de l'énonciateur, de ses stratégies de persuasion ou de séduction, des dispositifs argumentatifs, de leur questionnement : ces aspects émergent dans la tension locutoire entre l'explicite et l'implicite et peuvent être induits à la lecture du récit historique aussi bien que du récit médiatique, comme du reste, de tous les textes de communication et tous les genres discursifs. C'est pourquoi, en tant que linguiste, nous ne pouvons envisager l'événement médiatique qu'à travers la lecture et l'interprétation de sa mise en discours, en écartant les questions ontologiques. Nous avons eu parfois le sentiment que l'on voulait opérer une distinction entre les faits ou les événements en tant qu'objets du monde extralinguistique et leur occurrence en tant que signes linguistiques à l'œuvre dans le discours. Mais peut-on séparer ce dont nous parlons du comment nous en parlons? Les expressions « reconstruire des faits » et « interpréter un événement » sont des jeux de langage (au sens wittgensteinien), une manière socialement partagée d'attribuer une signification, qui ne peut valoir que pour « reconstruire la représentation des faits » ou « interpréter le récit d'un événement ». 


\section{BIBLIOGRAPHY}

AA.VV. 2002. Le style du Monde - Hors série du journal Le Monde (Paris : SA du Monde)

Amossy, Ruth. 2000. L'argumentation dans le discours (Paris : Nathan Université)

Amossy, Ruth. 2014. Apologie de la polémique (Paris : PUF)

Arquembourg, Jocelyne. 2003. Le temps des événements médiatiques (Paris : De Boeck-INA)

Arquembourg, Jocelyne. 2011. L'événement et les médias - Les récits médiatiques des tsunamis et les débats publics (1755-2004) (Paris : Archives contemporaines)

Azouzi, Ammar. 2013. «La "Révolution du jasmin" en Tunisie et son slogan "Ben Ali dégage !" : un événement discursif », Ballardini, Elio et al. (éds.). 2013

Baklouti, Elodie \& Arnaud Richard. 2013. «L'insulte à la Une d'un quotidien sportif : un événement médiatique », Ballardini, Elio et al. (éds.). 2013

Ballardini, Elio, Roberta Pederzoli, Sandrine Reboul-Touré \& Geneviève Tréguer-Felten (éds), Les facettes de l'événement : des formes aux signes, mediAzioni 15 [en linge : http:// mediazioni.sitlec.unibo.it, consulté le 1 mars 2015]

Ballardini, Elio. 2013. « Interpréter et traduire au procès Eichmann : construction médiatique d'un événement plurilingue », Londei, Danielle et al (éds), 161-169

Branca-Rosoff, Sonia, André Collinot, Jacques Guilhaumou \& Francine Mazière. 1995. « Questions d'histoire et de sens » Langages 115, 54-66

Branca-Rosoff, Sonia \& Jacques Guilhaumou. 1998. « De "société" à "socialisme" : l'invention néologique et son contexte discursif » Langage et société 83, 39-77

Calabrese, Laura. 2008. «Les héméronymes, ces événements qui font date, ces dates qui deviennent événements » Mots. Les langages du politique 88, 115-128

Calabrese, Laura. 2013a. L'événement en discours - Presse et mémoire sociale (Louvain-La-Neuve : Academia L'Harmattan)

Calabrese, Laura. 2013b. «La nomination des évènements dans le discours d'information : entre déférence et activité collective », Londei, Danielle et al (éds), 2013. 233-243

Charaudeau, Patrick. 2005. Les médias et l'information. L'impossible transparence du discours (Bruxelles : Duculot)

Charolles, Michel \& Béatrice Lamiroy. 2014. «"Fait” ou "événement" : quelles différences linguistiques? », Londei, Danielle et al (éds), 2013. 297-307

Douël, Jacques. 1987. Le journal tel qu'il est lu, (Paris : Centre de formation et de perfectionnement des journalistes)

Foucault, Michel. 1969. L'archéologie du savoir (Paris : Gallimard)

Foucault, Michel. 1971. L'ordre du discours (Paris : Gallimard)

Franckel, Jean-Jacques. 2002. « Le lexique entre identité et variation », Langue française 133, 3-15

Goetschel, Pascale \& Christophe Granger. 2011a. « Faire l'événement - Un enjeu des sociétés contemporaines ", Sociétés \& Représentations 32, 9-23 
Goetschel, Pascale \& Christophe Granger, 2011b. « "L'événement, c'est ce qui advient à ce qui est advenu..." - Entretien avec Pierre Laborie », Sociétés \& Représentations 32, 167-181

Guilhaumou, Jacques. 2006. Discours et événement - L'histoire langagière des concepts, (Besançon : Presses Universitaires de Franche-Comté)

Hagège, Claude. 1995. « Le rôle des médiaphoriques dans la langue et dans le discours ", Bulletin de la Société Linguistique de Paris, t.90 - fasc.1 \& 2, 1-19

Hailon, Fred. 2013. « L'événement-argument, support de la circulation idéologique : Villiers-leBel (nov.2007) et la "voyoucratie" ", Londei, Danielle et al. (éds). 2013. 253-266

Jamet, Claude. 2004. « La stratégie de l'évanouissement. À propos des tempêtes de décembre $1999 »$, Mots 75. 77-87

Kiefer, Ferenc. 1998. « Les substantifs déverbaux événementiels », Langages 131, 56-63

Koren, Roselyne. 1996. Les enjeux éthiques de l'écriture de presse (Paris : L'Harmattan)

Krieg-Planque, Alice. 2009. La notion de «formule » en analyse du discours. Cadre théorique et méthodologique (Besançon : Presses Universitaires de Franche-Comté)

Kripke, Saul. 1980. Naming and necessity (Oxford : Blackwell)

Lamizet, Bernard. 2013. «La sémiotique de l'événement : une sémiotique de l'espace et du temps », Ballardini, Elio et al. (éds.). 2013

Londei, Danielle, Sophie Moirand, Sandrine Reboul-Touré \& Licia Reggiani (éds). 2013. Dire l'événement. Langage, mémoire, société (Paris : Presse Sorbonne Nouvelle)

Maingueneau, Dominique. 2006. «Les énoncés détachés dans la presse écrite. De la surassertion à l'aphorisation », Bonhomme, Marc \& Gilles Lugrin (éds). Interdiscours et intertextualité dans les médias, TRANEL. Travaux Neuchâtelois de linguistique 44, 107-120

Margarito, Mariagrazia. 2013. "Marcher, courir : écriture et non-événement », Londei, Danielle et al. (éds). 2013. 285-293

Modena, Silvia. 2013. «Le "passage à l'euro" : quand la formule précède l'événement » Ballardini, Elio et al. (éds.). 2013

Moirand, Sophie. 2007a. Le discours dans la presse quotidienne : observer, analyser, comprendre (Paris : PUF)

Moirand, Sophie. 2007b. « Discours, mémoires et contextes : à propos du fonctionnement de l'allusion dans la presse », Corela 6 (hors série) [en ligne : http://corela.edel.univ-poitiers.fr/ index.php?id=1567, consulté le 1 mars 2015]

Nora, Pierre. 1972. «L'événement monstre », Communications 18. 162-172

Perelman, Chaïm, Lucie Olbrechts-Tyteca. 1992 (1958). Traité de l'argumentation (Bruxelles :

Éditions de l'Université libre de Bruxelles)

Picone, Michael. 1991. «L'impulsion synthétique : le français poussé par la synthèse », Le Français Moderne 59, 148-163

Puccinelli, Orlandi. 2013. «Événement discursif, mémoire et interprétation : à propos du documentaire Sao Carlos/1968 », Londei, Danielle, et al. (éds). 2013, 185-193

Quéré, Louis. 1994. "L'événement "sous une description" : contraintes sémantiques, croyances stéréotypiques et "natural facts of life as a morality" ", Prothée 22, 14-28

Quéré, Louis. 2006. « Entre fait et sens - La dualité de l'événement », Réseaux 139, 185-217 
Quéré, Louis. 2013. « Les formes de l'événement », Ballardini, Elio et al. (éds.). 2013

Rabattel, Alain. 2006. « Les autocitations et leurs reformulations: des surassertions surénoncées ou sousénoncées ", Travaux de linguistique 52, 71-84

Ravazzolo, Elisa. 2013. "Stratégies de représentation d'un “événement provoqué" - le débat sur l'interdiction du voile intégral à la télévision », Londei, Danielle et al. (éds). 2013, 87-97

Saussure, Ferdinand. 2002. Écrits de linguistique générale, Bouquet, Simon \& Rudolf Engler (éds)

(Paris : Gallimard)

Siblot, Paul. 2001. « De la dénomination à la nomination - Les dynamiques de la signifiance nominale et le propre du nom », Cahiers de praxématique 36, 1-26

Sitri, Frédérique. 2013. «Une lecture événementielle du verbe pouvoir dans des rapports de travailleurs sociaux ", Londei, Danielle et al. (éds). 2013. 73-83

Steiner, Georges. 1978. Après Babel - Une poétique du dire et de la traduction (Paris : Albin Michel)

Veniard, Marie. 2013. La nomination des événements dans la presse. Essai de sémantique discursive (Besançon : Presses universitaires de Franche-Comté)

Veron, Eliseo. 1981. Construire l'événement - les médias et l'accident de Three Miles Island (Paris : Éditions de Minuit)

\section{ABSTRACTS}

In this paper, we develop a linguistic reflection on the expression of the media event, from several recent essays that attempt to define the concept of "event", examined in the specific context of press discourse. We will first define specific uses in ordinary language of the terms "fait" and "événement", and then of the more precise concept of "media event", to which multidisciplinary studies are dedicated (historical, sociological, linguistic or information Science). The "media event" is a social and discursive construction. The study of its denomination, mainly in newspaper headlines, reveals lexical, syntactic, semantic and pragmatic features. However, we will propose a broader reflection on the enunciative or argumentative issues that are involved in the setting of the event story, a narration that is also a "putting into words" of the event.

À partir de plusieurs essais récents qui tentent de circonscrire le concept d'«événement», envisagé dans le cadre particulier des discours de la presse écrite, nous développons ici une réflexion linguistique autour de l'expression de l'événement médiatique. Nous définissons, dans un premier temps, les usages particuliers dans le langage ordinaire des deux termes "fait» et "événement» avant de nous attarder sur le concept plus précis d'«événement médiatique », auquel ont été consacrées des études pluridisciplinaires (historiques, sociologiques, linguistiques, sciences de l'information). L'événement médiatique est le résultat d'une construction sociale et discursive. L'étude de sa dénomination, essentiellement dans les titres de journaux, relève d'aspects à la fois lexicologiques, syntaxiques ou sémanticopragmatiques. Mais nous proposons également une réflexion plus étendue sur les problématiques énonciatives ou argumentatives qui entrent en jeu dans la mise en récit de l'événement, une narration qui est aussi une mise en discours. 
INDEX

Mots-clés: discours, événement, médias

Keywords: discourse, event, media

\section{AUTHOR}

LORELLA SINI

Università di Pisa 